within the sequences of all larger RNAs; the $3^{\prime}$-termini of all the RNAs are common, and they all extend inward from the $3^{\prime}$ end of the genome. In vitro translation experiments by S. Siddell (Würzburg) and B. van der Zeijst (Utrecht) showed that each subgenomic RNA is a messenger species which specifies a single primary protein product translated from its unique $5^{\prime}$-terminal sequence. Nothing is known at present as to the mechanism by which these subgenomic RNAs are synthesised, although UV target size measurements suggest that each is independently initiated on a negative strand template, and not derived by processing from a larger species (van der Zeijst). A membrane-bound RNA-dependent RNA polymerase activity present in cells infected with a porcine coronavirus was described by $\mathrm{D}$. Brian (Knoxville), but as yet no evidence as to the nature of replicative intermediate molecules has been obtained.

Of the three well-defined virion proteins, one is a $60 \mathrm{~K}$ nucleocapsid protein phosphorylated by a virion-bound protein kinase, and the others are envelope glycoproteins. The largest $(90 \mathrm{~K})$ forms the petallike structures (peplomers) of the 'crown' (from which the group derives its names), and apparently has a conventional mode of synthesis similar to the glycoproteins of other enveloped viruses. The smaller glycoprotein $(23 \mathrm{~K})$ is embedded in the envelope lipid bilayer, and is largely unaltered by treatment of the virus particle with bromelain, which removes the peplomers; this protein thus fulfils a similar role to that of the membrane protein of other enveloped viruses such as influenza. Glycosylation of this coronavirus membrane protein has unique features. $R$. Rott (Giessen) showed for a bovine coronavirus that the $23 \mathrm{~K}$ protein contains no fucose or mannose. $\mathrm{H}$. Niemann and $\mathrm{H}$. Klenk (Giessen), P. Rottier (Utrecht) and $\mathrm{K}$. Holmes (Bethesda) all reported that synthesis of the $23 \mathrm{~K}$ glycoprotein is not inhibited by tunicamycin; this indicates that, in contrast to all other virus glycoproteins studied so far (including the $90 \mathrm{~K}$ corona-virus protein) dolichol-linked $\mathrm{N}$-acetylglucosamine plays no part in biosynthesis. L. Sturman (Albany) showed that in the presence of tunicamycin virus particles still matured, but were released from the cells as 'spikeless' virions lacking the $90 \mathrm{~K}$ protein.

Comparisons between the genomic and subgenomic RNAs of coronaviruses differing in pathogenic potential or species of origin were reported by several workers. $T_{1}$ ribonuclease mapping studies by $B$. Lomniczi (Budapest) gave rise to at least 11 different genome RNA fingerprints within 13 isolates of avian coronavirus orginally classified as infectious bronchitis by pathological criteria. However it is likely that this technique provides too fine an analysis of genome structure for useful strain comparisons. S. Weiss (Philadelphia) described the in vitro

\title{
Molecular lines in the red
}

\section{from a correspondent}

THE FIRST OBSERVATIONS of molecular emission in the far infra-red (IR) from an interstellar cloud have been made by a team at the University of California (D.W. Watson, J.W.V. Storey, C.H. Townes, E.E. Haller and W.L. Hansen Astrophys. J. 239, L129; 1980). These observations were made from the NASA Kuiper Airborn Observatory in January 1980 at an altitude of $12.5 \mathrm{~km}$, using a liquid helium-cooled $\mathrm{Ge}: \mathrm{Sb}$ photodetector in a metal-mesh, scanning Fabry-Pérot interferometer. The two $J$ $=21 \rightarrow 20$ and $J=22 \rightarrow 21$ rotational lines of $\mathrm{CO}$ were observed in emission at 124 and $119 \mu \mathrm{m}$ from the KleinmannLow region of the Orion molecular cloud. The CO molecule in interstellar clouds has previously been observed in the millimetre and submillimetre regions of the spectrum, where it has been shown to be one of the principal molecular species containing carbon in dense molecular clouds such as Orion. The significance of the new $124-$ and $119-\mu \mathrm{m}$ observations is that for these rotational transitions to be observable at all, the $\mathrm{CO}$ must be much

synthesis of a representative DNA copy of the genome of MHV strain A59. Using this cDNA probe, she found considerable sequence homology with other murine coronaviruses and a low level of homology with $229 \mathrm{E}$ virus, a human coronavirus. Extension of such studies to the virus isolates from multiple sclerosis brain tissue, which were obtained by passage of the tissue in mice, should help to identify the origin of these agents.

The second half of the meeting was devoted to persistency and pathogenesis. Although little is known of the molecular mechanisms underlying organotropism and pathogenesis following coronavirus infection, some advances were reported. Coronavirus-induced disease is very dependent upon the age at infection, genetic background, and route of inoculation of the host. K. Pickel (Würzburg) showed that mice were fully susceptible to fatal JHM virus infection up to the age of 21 days, but thereafter became fully resistant. Resistance could be induced by transfer of immune spleen cells from adult mice to baby mice, or by priming of adult non-immune spleen cells in baby mice with UV-inactivated JHM virus, but not by non-immune spleen cells alone. R. Knobler (La Jolla) confirmed that fatal encephalomyelitis induced by JHM virus is an autosomal dominant trait; the ability of the virus to induce demyelinating lesions hotter $(500-1,000 \mathrm{~K})$ than the temperature of the $\mathrm{CO}$ molecules observed in the microwave $(\sim 100 \mathrm{~K})$. These high temperatures indicate that the $\mathrm{CO}$ observed in the far IR is present in a post-shock gas, corroborating earlier evidence obtained from $\mathrm{H}_{2}$ observations that a shock wave is propagating into the Orion cloud.

This observational technology opens up an entire new spectral range for observations of molecular species in the interstellar medium. Until now observations of interstellar clouds have been limited mainly to the microwave and radio regions at the long-wavelength end of the spectrum, and to the visible and near ultra-violet regions at the short end of the spectrum. The IR region of the spectrum is a rich arena for remotesensing applications, but it has been essentially closed to interstellar observations by a combination of detector problems and atmospheric absorption. The development of far-IR techniques for observation of the interstellar medium increases the potential for new discoveries concerning the structure, dynamics, chemistry and evolution of interstellar clouds.

depended upon the cell tropism of selected mutants to replicate in oligodendroglial cells. H. Wege (Würzburg) reported that injection of JHM ts mutants into preimmunised rats by the intracerebral route causes a high rate of subacute to chronic demyelinating disease with similarities to virus-induced demyelination in man. Rats survive normally after intraperitoneal injection of similar virus doses.

Several investigators have studied the mechanism of resistance at the cellular level. F. Bang (Baltimore), who had shown as early as 1960 that peritoneal macrophages taken from MHV resistant mice were also resistant to infection in vitro, now reported that macrophage resistance is also dependent upon associated lymphocyte action and may also involve interferon. Extension of these studies from macrophages to primary mouse hepatocyte monolayer cultures by H. Arnheiter (Zürich) confirmed that the hepatocytes were genetically resistant to virus infection as were the mice themselves. Although interferon treatment reduced virus titres in susceptible cell cultures, addition of anti-interferon antiserum did not augment the susceptibility of resistant hepatocyte cultures. Thus it can be concluded that such cells are intrinsically resistant to virus infection, though by what mechanism remains to be determined. 